\title{
Tumor-Derived Soluble MICA Obstructs the NKG2D Pathway to Restrain NK Cytotoxicity
}

\author{
Qizhi Luo', Weiguang Luo ${ }^{1,2}$, Quan Zhu' ${ }^{1}$, Hongjun Huang ${ }^{3}$, Huiyun Peng ${ }^{1}$, Rongjiao Liu ${ }^{1}$, Min \\ $\mathrm{Xie}^{1}$, Shili $\mathrm{Li}^{1}$, Ming Li ${ }^{1}$, Xiaocui Hu${ }^{3}$, Yizhou Zou, ${ }^{1, *}$ \\ ${ }^{1}$ Department of Immunology, Basic Medical School of Central South University, Changsha, Hunan, China. \\ ${ }^{2}$ Department of Physiology, University of Texas Southwestern Medical Center at Dallas, TX, USA \\ ${ }^{3}$ Cancer Hospital of Hunan, Xiangya Medical School, Central South University, Changsha, Hunan, China.
}

[Received May 15, 2019; Revised June 17, 2019; Accepted June 28, 2019]

\begin{abstract}
The natural killer group 2D (NKG2D) receptor and its ligands play important roles in immune surveillance. In this study, we observed that the average serum soluble MICA （sMICA） concentration of 174 hepatocellular carcinoma (HCC) patients was significantly higher than that in 80 healthy subjects $(602.17 \pm$ 338.15 vs. $72.26 \pm 87.88 \mathrm{pg} / \mathrm{ml}, \mathrm{t}=3.107, \mathrm{P}=\mathbf{0 . 0 0 2}$ ). The levels of serum sMICA in $44 \mathrm{HCC}$ patients with initial levels above $400 \mathrm{pg} / \mathrm{ml}$ declined significantly after surgical removal of the liver cancer tissue $(P<0.001)$. Moreover, the mean survival time of HCC patients who had sMICA above $400 \mathrm{pg} / \mathrm{ml}$ was significantly shorter than that HCC patients with lower sMICA levels $(P<0.001)$. Using the reporter cell line (NKG2D-2B4) in which activation of the NKG2D receptor pathway results in GFP expression based on the stimulation of immobilized rMICA, we showed that the number of GFP-expressing cells decreased sharply in presence of sMICA. Upon adding sMICA, the release of cytokines IFN- $\gamma$, TNF- $\alpha$, and IL-8 by NK cell line (NKL) under stimulation of immobilized rMICA was blocked. Using MICA-expressing cells as the target cells, we observed that about $80 \%$ of target cells were killed by NKL at E:T of 10:1, but in presence of sMICA ${ }^{\text {high }}$ serum of HCC patients, the dead target cells were reduced to $30.8 \%$. Compared in presence of sMICA $^{\text {low }}$ serum from HCC patients, there were 63.7\% of target cells dead $(p=0.043)$. Thus, our data suggested that sMICA obstructs the activation of NKG2D pathway to protect tumor cells from NK cell-mediated cytotoxicity.
\end{abstract}

Key words: hepatocellular carcinoma (HCC), NKG2D, soluble MICA, NKG2D receptor pathway, tumor immune escape

Natural killer (NK) cells play an important role in immune surveillance for viruses and tumor cells in a way that is independent from antigen presentation by major histocompatibility complex (MHC) molecules [1, 2]. There are many inhibitory and activating receptors on the surface of NK cells; NKG2D [3], an important activated receptor, belongs to the type II transmembrane type $\mathrm{C}$ lectin-like receptor family [4-6]. Binding to NKG2D activates the NKG2D pathway and triggers cytotoxic activities of $\mathrm{NK}$ and $\mathrm{CD} 8^{+} \mathrm{T}$ cells against target cells [7].
The NKG2D receptor ligands include the major histcompatibility complex class I related chains A and B (MICA and MICB, respectively) and UL16 binding proteins 1-6 (ULBP1-6) [8]. MICA and MICB are expressed in gastrointestinal epithelial cells and fibroblasts at low levels under physiological conditions [9]. Upon infection or in the presence of a tumor, the expression of MICA and MICB increased [10]. We previously reported that human fibroblasts that overexpressed MICA were more sensitive to NK cells

*Correspondence should be addressed to: Dr. Yizhou Zou, Department of Immunology, Basic Medical School of Central South University, Changsha, Hunan, China. Email: yizhou_zou@sina.com.

Copyright: () 2019 Luo Q et al. This is an open-access article distributed under the terms of the Creative Commons Attribution License, which permits unrestricted use, distribution, and reproduction in any medium, provided the original author and source are credited. 
than normal fibroblasts [11]. Furthermore, we discovered that human cytomegalovirus infection leaded to a decrease in the expression of MICA on the fibroblast surface, which allowed these virally infected cells to resist NK cell-mediated cytotoxicity. The reduction of NK cellmediate killing during viral infection and tumor genesis is associated with a decrease in levels of the NKG2D receptor and NKG2D receptor ligands [12].

MICA expression is regulated under stress conditions [13]. When MICA binds to the NKG2D receptor of NK cell, the associating membrane molecules DAP10 are phosphorylated to recruit and activate PI3K downstream pathway, resulting in MICA-expressing target cell killing upon NK cell activation [14-16]. Tumor cells have evolved a variety of immune escape mechanisms. Soluble NKG2D ligand molecules that block NKG2D activation were observed in patient with a variety of solid tumor types [17]. For example, tumor cells release soluble MICA (sMICA) by proteolytic shedding off from membrane-associated MICA [18, 19]. In osteosarcomas cells, it is MMP9 that hydrolyzes MICA [20]. In glioblastoma cell lines, ADAM10 and ADAM17 hydrolyze ULBP2 to form another soluble NKG2D receptor ligand [21]. Jiang et al. found that certain MICA gene polymorphisms are associated with survival in hepatocellular carcinoma (HCC) patients [22]. HCC tumor cells shed sMICA [23]. It has been proposed that sMICA can specifically bind to the NKG2D receptor without activating NKG2D-mediated signaling. Moreover, sMICA binding may prevent the NKG2D receptor from contacting NKG2D receptor ligands on the tumor cell surface, which may allow tumor immune escape [24].

In our previous study, we showed that a fusion protein composed of the MICA extracellular domain (MICA$\mathrm{ECD})$ and an anti-CD20 single-chain antibody ( $\mathrm{ScFv}$ ) induced NK cell-mediated killing of CD20 tumor cells [25]. NKG2D and its ligands are involved in the development of prostate cancer, leukemia, melanoma, and other tumors [26]. Others have suggested the potential of immunotherapies based on the NKG2D pathway [27]. Here we investigated how serum sMICA levels in HCC patients are correlated with survival and studied how sMICA influences the activation of the NKG2D pathway using an NKG2D receptor reporter cell line.

\section{MATERIALS AND METHODS}

\section{HCC patient samples}

Blood samples were drawn from 174 patients diagnosed with HCC in Hunan Cancer Hospital from January 2017 to December 2018. The cohort included 137 males and 37 females with an average age of $55 \pm 13$ years. All HCC patients were diagnosed based on examination of tissue by pathologists and other clinic diagnosis. The control group included 80 healthy volunteers $(62$ males and 18 females) with an average age of $56 \pm 12$ years. All participants in this study provided informed consent. This study was approved by the ethics committee of Hunan Cancer Hospital and conducted in strict accordance with the requirements of the ethics committee.

\section{Cells and cell culture}

Human fibroblasts cell (HFC), T hybridoma 2B4 cells, and the human NKG2D reporter cells (NKG2D-2B4 cells), which have been prepared as our previous work [25], were cultured in RPMI-1640 medium with $10 \%$ FBS at $37{ }^{\circ} \mathrm{C}$ in $5 \% \mathrm{CO}_{2}$. The NKG2D reporter cells were constructed NKG2D receptor on $2 \mathrm{~B} 4$ cells containing a nuclear factor of activated $\mathrm{T}$ cells (NFAT)-responsive green fluorescent protein (GFP) reporter gene [28]. The engagement of the NKG2D receptor induces the activation of NFAT and expression of GFP. The NK cell line (NKL, provided by M. J. Robertson, Indiana University School of Medicine, Indianapolis, IN) was cultured in RPMI-1640 medium with $15 \%$ FBS and 10 $\mathrm{ng} / \mathrm{mL}$ of recombinant IL-2 (Sino Biological Inc.) at 37 ${ }^{\circ} \mathrm{C}$ in $5 \% \mathrm{CO}_{2}$. MICA stable expressed human fibroblast cells $\left(\mathrm{MICA}^{+} \mathrm{HFC}\right)$ were prepared by transfection of the vector pCDNA3.1 (-) inserting with the cDNA encoding full-length MICA and were selected with $100 \mu \mathrm{g} / \mathrm{ml} \mathrm{G} 418$ in culture medium.

\section{Preparation of recombinant MICA and NKG2D-Ig}

HEK293T cells $\left(1 \times 10^{6}\right.$ cells $)$ were added to $10 \mathrm{ml}$ DMEM, and the cells were cultured at 85\% 90\% confluence. At 1 hour before transfection, $5 \mathrm{ml}$ of complete culture medium without antibiotics was added. Constructed vectors with codons of MICA*004 extracellular domains fused to a His-tag and vectors with codons of NKG2D extracellular domains fused to a human IgG-Fc and a His-tag were added, then culturing for 72 hours and collecting the culture media. The media were centrifuged at $10000 \mathrm{rpm}$ for 5 minutes, and supernatant was collected and stored at $-80{ }^{\circ} \mathrm{C}$ if needed, The supernatant contained the recombinant proteins of

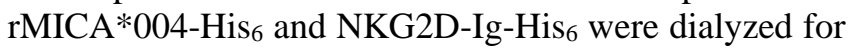
5 days against Tris-HCl buffer ( $\mathrm{pH} 7.5$ ). The buffers were changed with new one every 12 hours. After dialysis, $\mathrm{NaCl}$ and imidazole were added to final concentrations of $500 \mathrm{mM}$ and $20 \mathrm{mM}$, respectively. Ni-NTA protein purification kit ( $\mathrm{pH}=6.8-7.2$, Konipa, Guangzhou, China) was used to purify the His-tag fusion proteins in solution according the instruction of manufactory. 


\section{Sandwich ELISA to detect SMICA}

Anti-MICA antibody (AMO1, Immatics; mouse IgG1, $5.0 \mu \mathrm{g} / \mathrm{ml})$ was coated to reaction wells $(100 \mu l$ per well $)$ at $4^{\circ} \mathrm{C}$ over night, and after washed with PBS containing $0.05 \%$ Tween-20, $100 \mu \mathrm{l}$ the blocking buffer (PBS containing $5 \%$ BSA) were added and incubated at $37^{\circ} \mathrm{C}$ for $2 \mathrm{~h}$. Then after removal of blocking buffer, washed the well with washing buffer (PBS containing $0.05 \%$ Tween20) for two times. Next, rMICA*004 was diluted to prepare eight standard solutions within the range of 100 to $6309 \mathrm{pg} / \mathrm{ml}$. Standard solutions, serum samples were added into each well, respectively; after incubated at 37 ${ }^{\circ} \mathrm{C}$ for 2 hour, wells were washed with $200 \mu$ l of washing buffer for four times. Then $100 \mu 1$ of MICA-specific monoclonal antibody (6B3) [29], mouse IgG2a, $1.0 \mu \mathrm{g} / \mathrm{ml}$ diluted with PBS containing $3.75 \%$ BSA) was added to each well. After incubation at $37^{\circ} \mathrm{C}$ for $2 \mathrm{~h}$, wells were washed with washing buffer for four times, then, $100 \mu 1$ HRP-conjugated goat anti-mouse IgG2a antibodies (BD Biosciences) diluted with PBS containing $3.75 \%$ BSA at 1:10000 ratio was added to each well and incubated at 37 ${ }^{\circ} \mathrm{C}$ for $1 \mathrm{~h}$. After washed with washing buffer for four times, $100 \mu$ l of chromic substrate (tetramethyl benzidine) was added to each well, and the reaction was terminated with $4 \mathrm{~N} \mathrm{H}_{2} \mathrm{SO}_{4}$ after incubation at $37^{\circ} \mathrm{C}$ for $15 \mathrm{~min}$. The OD value was detected at the wavelength of $450 \mathrm{~nm}$, and sMICA in serum was quantified by comparison to the standard curve generated by analysis of the recombinant protein.

\section{Flow cytometry}

The level of GFP-expressing NKG2D-2B4 reporter cells was determined by flow cytometry. The rMICA and control BSA at $50 \mu \mathrm{g} / \mathrm{ml}$ were added to each well of a 96well plate in a $50 \mu \mathrm{l}$ volume, respectively. To perform as the immobilized ligand of NKG2D receptor (immobilezed-rMICA) and allow rMICA adhere to the well, the soluble rMICA was added to wells and incubated at $37^{\circ} \mathrm{C}$ for $4 \mathrm{~h}$. After washed with PBS, the concentration of NKG2D-2B 4 cells was adjusted to $4 \times 10^{5}$ cells $/ \mathrm{ml}$ with RPMI-1640 medium containing 10\% FBS, and $200 \mu \mathrm{l}$ of this cell suspension was added to each well. GFP generated under NKG2D receptor activation was detected by flow cytometry after incubation at $37^{\circ} \mathrm{C}$ for $16 \mathrm{~h}$. The degree of activation of the NKG2D signaling pathway was determined based on the percentage of $\mathrm{GFP}^{+}$cells. Anti-NKG2D antibody (1:2000, Thermo Fisher Scientific) and anti-His tag antibody (1:1000, Bio-Rad) were used for detection of NKG2D expression and the binding of his-rMICA*004 to NKG2D receptors of cells.

Meanwhile, $2 \times 10^{6}$ NKG2D-2B4 reporter cells were collected, centrifuged at $800 \mathrm{rpm}$ for $5 \mathrm{~min}$, washed with
PBS containing 2\% FBS, and then re-suspended in PBS. Subsequently, w6/32 (5 $\mu \mathrm{g} / \mathrm{ml}$, ATCC, USA) was added, anti-NKG2D antibody was added at 1:200, and antiMICA antibody was added at 1:200 (antibody concentrations were $5 \mu \mathrm{g} / \mathrm{ml}$ ). After incubation at $4{ }^{\circ} \mathrm{C}$ for $30 \mathrm{~min}, 2 \%$ FBS-PBS was added, and then anti-mouse IgG FITC monoclonal antibody (BD Pharmingen, 1:400) was added and incubated at $4{ }^{\circ} \mathrm{C}$ for $30 \mathrm{~min}$. The cells were re-suspended with $500 \mu \mathrm{l} 2 \%$ FBS-PBS and analyzed by flow cytometry (Athena dxp, Cytek Company).

\section{Absorption of SMICA from serum}

$50 \mu \mathrm{g} / \mathrm{ml}$ stock of anti-MICA specific monoclonal antibodies $6 \mathrm{~B} 3$ (mAb-6B3) was coated to the protein A micro-magnet beads (Kangsheng Biotechnology, Guangzhou, China). After incubated at $37^{\circ} \mathrm{C}$ for $8 \mathrm{~h}$. The supernatant was discarded, and the pellet was washed with PBS three times, HCC patient serum, diluted 1:3 in PBS (pH7.5) was added into 6B3-beads prepared, and incubated at $37{ }^{\circ} \mathrm{C}$ for $2 \mathrm{~h}$. Then, repeated above absorption procedure. Finally, the treated samples were collected and saved in $-80{ }^{\circ} \mathrm{C}$ until use. In addition, the same serum sample was proceeded the sMICA absorption by uncoaded beads and used as control.

\section{NK cell cytotoxic activity}

Target cells of human fibroblasts with or without stable expression of $\mathrm{MICA}^{*} 027$ by viral transduction were collected by centrifugation at $800 \mathrm{rpm}$ for $5 \mathrm{~min}$. After washing with sterile PBS, cells were centrifuged at 800 $\mathrm{rpm}$ for $5 \mathrm{~min}$. The target cells were suspended in sterile PBS to $1 \times 10^{6}$ cells $/ \mathrm{ml}$. CFSE (Sigma) was added into the cells at a final concentration of $200 \mathrm{nM}$ in PBS, and cells were stained at $37{ }^{\circ} \mathrm{C}$ for $20 \mathrm{~min}$. After washing three times with PBS, the cells were transferred into wells of a 96-well plate at 5000 cells per well. NK cells were added to the wells with the target cells stained with CSFE at a ratio (E:T) of 1:1, 5:1, 10:1, and 20:1 in a final volume of $200 \mu \mathrm{l}$. After mixing, the cells were centrifuged at 800 rpm for $1 \mathrm{~min}$ and incubated at $37{ }^{\circ} \mathrm{C}$ for $4 \mathrm{~h}$. After centrifugation at $2000 \mathrm{rpm}$ for $2 \mathrm{~min}$, the cells were collected and washed twice with PBS supplemented with 2\% FBS, stained with 7-AAD (7-Amino Actinomycin D, $\mathrm{BD}$ Pharmingen ${ }^{\mathrm{TM}}$ ) at room temperature for 10 minutes. In NKG2D pathway blocking assays, purifed rMICA diluted at concentrations among $0.1 \mathrm{ng} / \mathrm{ml}$ to $8.0 \mathrm{ng} / \mathrm{ml}$, sMICA $^{\text {high }}$ sera from HCC patients (sMICA $>400 \mathrm{pg} / \mathrm{ml}$, $\mathrm{n}=10$ ), sMICA $^{\text {low }}$ sera from HCC patients (sMICA $\leq 400$ $\mathrm{pg} / \mathrm{ml}, \mathrm{n}=10$ ), normal health serum and soluble NKG2DIg at concentration above $1.0 \mathrm{ug} / \mathrm{ml}$ were added to the mixture of target cells with NKL to block NKL killing, 
respectively. After mixing, the cells were centrifuged at $800 \mathrm{rpm}$ for $1 \mathrm{~min}$ and incubated at $37{ }^{\circ} \mathrm{C}$ for $4 \mathrm{~h}$. After centrifugation at $2000 \mathrm{rpm}$ for $2 \mathrm{~min}$, the cells were stained with 7-AAD. After washing with PBS, cells were fixed with $1 \%$ paraformaldehyde in $2 \%$ FBS and $0.1 \%$ $\mathrm{NaN}_{3}$ PBS buffer; the dead cells were analyzed by flow cytometry.

\section{NKG2D pathway activation and blocking}

rMICA at range of concentration was added to wells of a 96-well plate, allowing rMICA to adhere to the well of plate and incubated at $37{ }^{\circ} \mathrm{C}$ for 8 hours. Un-coated rMICA in the solution was discarded. NKG2D-2B4 cells were added to each well at a range of concentrations. After the cells were incubated for $24 \mathrm{~h}$ at $37^{\circ} \mathrm{C}$, the percentage of fluorescent cells was determined by flow cytometry. The concentration which generated the maximal number of fluorescent cells $\left(\mathrm{GFP}^{+}\right)$was used in subsequent experiments.

For analysis of pathway activation and pathway inhibition in the presence of serum, rMICA at a final concentration of $50 \mu \mathrm{g} / \mathrm{ml}$ was coated to wells of a 96well plate for NKG2D receptor activation. The solution was discarded after $8 \mathrm{~h}$ at $37{ }^{\circ} \mathrm{C}$. Aliquots of $100 \mu \mathrm{l}$ of NKG2D-2B4 cells at $1 \times 10^{6}$ cells $/ \mathrm{ml}$ were added to the tubes containing soluble rMICA, normal human sera, patient sera or soluble NKG2D-Ig fusion protein, and incubated at $37^{\circ} \mathrm{C}$ for $2 \mathrm{~h}$. Then aliquots of $100 \mu \mathrm{l}$ of treated and untreated cells were added to wells of 96-well plates with pre-coated rMICA (immobilized rMICA). After mixed, the cells were incubated at $37{ }^{\circ} \mathrm{C}$ for 4 hours. The proportion of cells with fluorescence was detected under a fluorescence microscope and analyzed with flow cytometry (Athena dxp, Cytek Company).

\section{Cytokine detection}

The cytokine Grp I group 17-plex kit (Bio-Rad) was used for this experiment. The analysis was performed according to the manufacturer's instructions using $50 \mu \mathrm{l}$ sample, standard, and buffer control. Samples were added into mixed Luminex array beads to incubation for 2 hours at room temperature. After washed, $25 \mu 1$ detection antibody was added and incubated at room temperature for $30 \mathrm{~min}$. After washed for 3 times, PE-Streptomyces anti-biotin protein $(50 \mu \mathrm{l})$ was added and incubated at room temperature for $10 \mathrm{~min}$. After washed, $125 \mu \mathrm{l}$ testbuffer was added to re-suspend the beads and analyzed using Luminex machine 200.

\section{Statistical analysis}

SPSS17.0 software was used to analyze the obtained data. Means \pm standard deviations are reported. Experiments were carried out independently three times. The twosample t test was used for statistical analysis, and the survival rate was analyzed by log-rank test. $\mathrm{P}<0.05$ was considered statistically significant.

\section{RESULTS}

\section{High serum levels of soluble MICA are associated with poor HCC prognosis}

Increased release of soluble MICA into circulation is common in patients with solid tumors. In order to detect and analyze the presence of sMICA in peripheral blood of HCC patients, we established a dual-antibody sandwich ELISA method to quantitatively determine the concentration of sMICA in serum. A standard curve was prepared by analysis of known concentrations of highly purified recombinant ( $\left.\mathrm{rMICA}^{*} 004\right)$ (Fig. 1A). The range of sMICA concentration that could be accurately detected was 100 to $6309 \mathrm{pg} / \mathrm{ml}$. Any serum samples that initially tested above $6309 \mathrm{pg} / \mathrm{ml}$ were retested after appropriate dilution.

The concentrations of sMICA in serum of healthy controls $(\mathrm{N}=80)$, HCC patients $(\mathrm{N}=174)$, and HCC patients' post-surgery $(\mathrm{N}=44)$ were measured with the sandwich ELISA. The mean concentrations of serum sMICA were $72.26 \pm 87.88,602.17 \pm 338.15$, and $301.62 \pm$ $288.20 \mathrm{pg} / \mathrm{ml}$, respectively (Fig. 1B). The serum sMICA concentration of HCC patients was significantly higher than that of the controls $(\mathrm{t}=3.107, \mathrm{P}=0.002)$. Of $80 \mathrm{HCC}$ patients, 44 with serum sMICA concentrations that exceeded $400 \mathrm{pg} / \mathrm{ml}$ were evaluated 15 days after resection of the liver tumor tissue. In these HCC patients, sMICA serum levels declined significantly post-surgery (1205.4 \pm $740.5 \mathrm{pg} / \mathrm{ml}$ vs. $701.2 \pm 843.5 \mathrm{pg} / \mathrm{ml}, \mathrm{t}=9.67, \mathrm{P}<0.001)$, Furthermore, pair analysis of HCC cases before and after surgery showed that the concentration of serum sMICA decreased in each patient after the liver cancer tissue was removed ( $\mathrm{t}=15.28, \mathrm{P}<0.001$, Fig. 1C).

HCC patients who were not treated surgically were divided into two groups based on serum sMICA concentrations: the sMICA ${ }^{\text {high }}$ group $(\mathrm{N}=60)$ had sMICA levels greater than or equal to $400 \mathrm{pg} / \mathrm{ml}$, and the sMICA $^{\text {low }}$ group $(\mathrm{N}=70)$ had sMICA levels below 400 $\mathrm{pg} / \mathrm{ml}$. Mean survival of patients in sMICA ${ }^{\text {high }}$ group was shorter than that of patients in the sMICA ${ }^{\text {low }}$ group (5.3 months vs. 8.8 months, Fig. 1D). This difference was highly significant $\left(\chi^{2}=81.988, \mathrm{P}<0.001\right)$, suggesting that the concentration of sMICA in the blood is correlated with prognosis. 

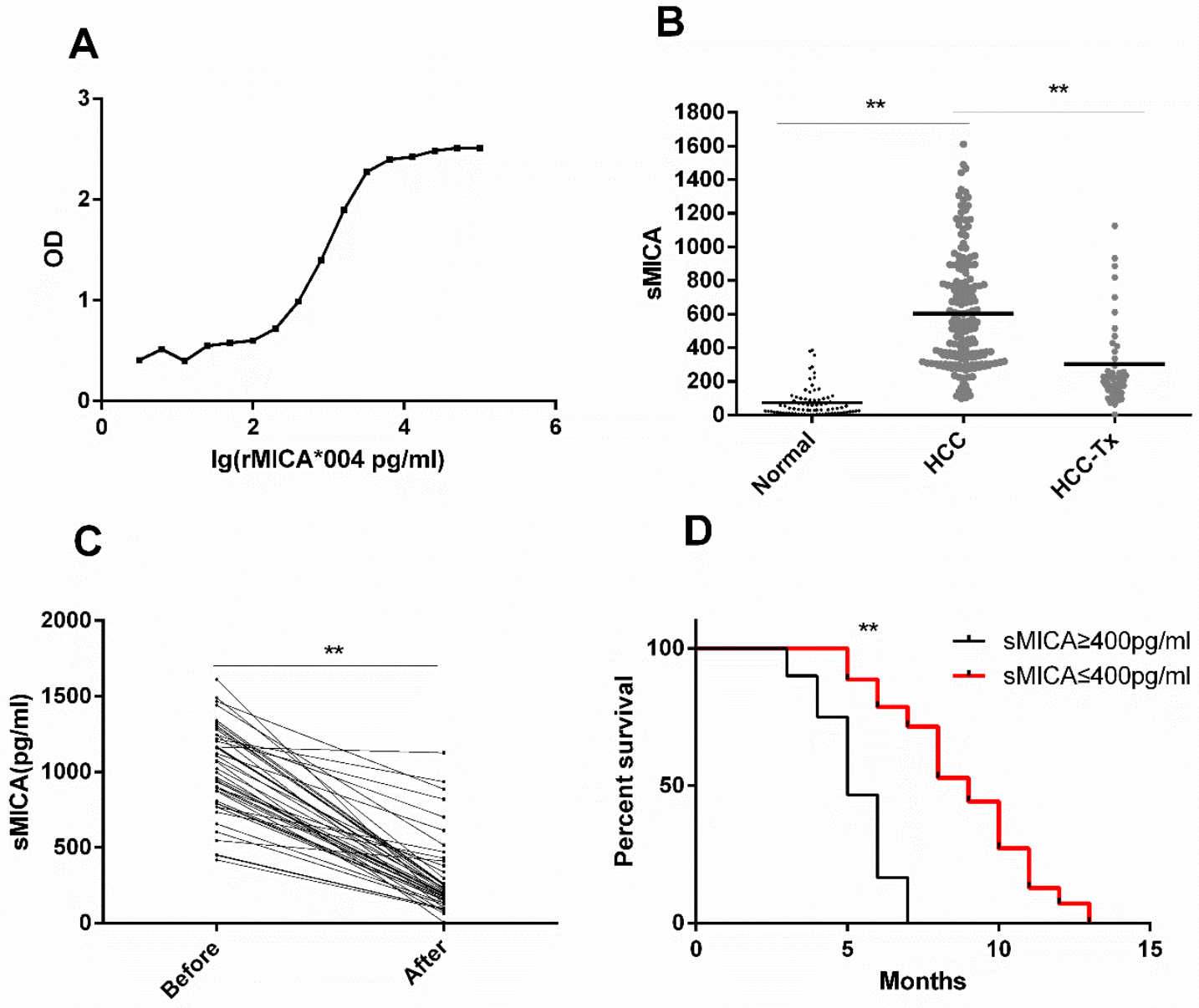

D

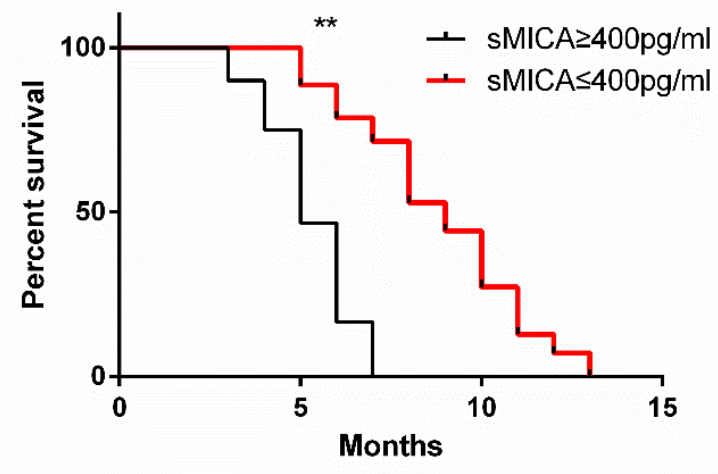

Figure 1. Serum sMICA concentration is associated with poor prognosis in HCC. (A) Standard curve for quantitative detection of sMICA using double-antibody sandwich ELISA made using purified rMICA*004 at eight concentrations ranging from 100 to $10000 \mathrm{pg} / \mathrm{ml}$. Plotted is OD vs. $\lg [\mathrm{rMICA}]$. (B) sMICA concentrations (pg/ml) in serum of healthy controls ( $\mathrm{N}=80)$, HCC patients $(\mathrm{N}=174)$, and $\mathrm{HCC}$ patients' post-surgical resection of tumors $(\mathrm{N}=44)$. The horizontal lines indicate means. ** Indicates $\mathrm{P}<0.05$. (C) Serum sMICA levels before and after surgery in HCC patients with serum sMICA $\geq 400 \mathrm{pg} / \mathrm{ml}$ before treatment $(\mathrm{N}=44)$. Serum samples were taken immediately prior to surgery and 15 days after surgery. Lines pair points from individual patients. ** Indicates $\mathrm{P}<0.05$. (D) Survival curves of HCC patients not treated surgically divided based on serum sMICA concentration: $\mathrm{sMICA}^{\text {low }}<400 \mathrm{pg} / \mathrm{ml}\left(\mathrm{N}=70\right.$, red) and $\mathrm{sMICA}^{\text {high }} \geq 400 \mathrm{pg} / \mathrm{ml}(\mathrm{N}=60$, black). Log-rank statistical analysis was performed on the survival curves between the two groups; ** Indicates $\mathrm{P}<0.05$.

\section{Establishment of NKG2D signaling evaluation system}

In order to determine whether sMICA influences signaling through the NKG2D receptor, we generated a combined NKG2D receptor cell model. We fused the extracellular domain of the human NKG2D to the transmembrane domain and cytoplasm tail of mouse NKG2D and expressed this construct in 2B4 cells (NKG2D-2B4 cells). This hybrid NKG2D receptor can bind NKG2D ligands (e.g., MICA) and activates signaling through the ITAM of the adapter membrane molecule DAP12 and the phosphorylation of Syk to activate transcription of GFP (Fig. 2A).
Since our data showed that the immobilized rMICA activated the NKG2D signaling pathway but not soluble sMICA, we used an anti-NKG2D monoclonal antibody to demonstrate the expression of NKG2D receptor on the NKG2D-2B4 cell surface and used an anti-His antibody to evaluate the binding of soluble MICA to the NKG2D2B4 cells. High levels of NKG2D receptors were detected on the NKG2D-2B4 cell surface, and the soluble rMICA did bind to these cells (Fig. 2B). This suggests that although soluble rMICA did not activate NKG2D receptor-mediated signaling, it does bind to the NKG2D receptor. 


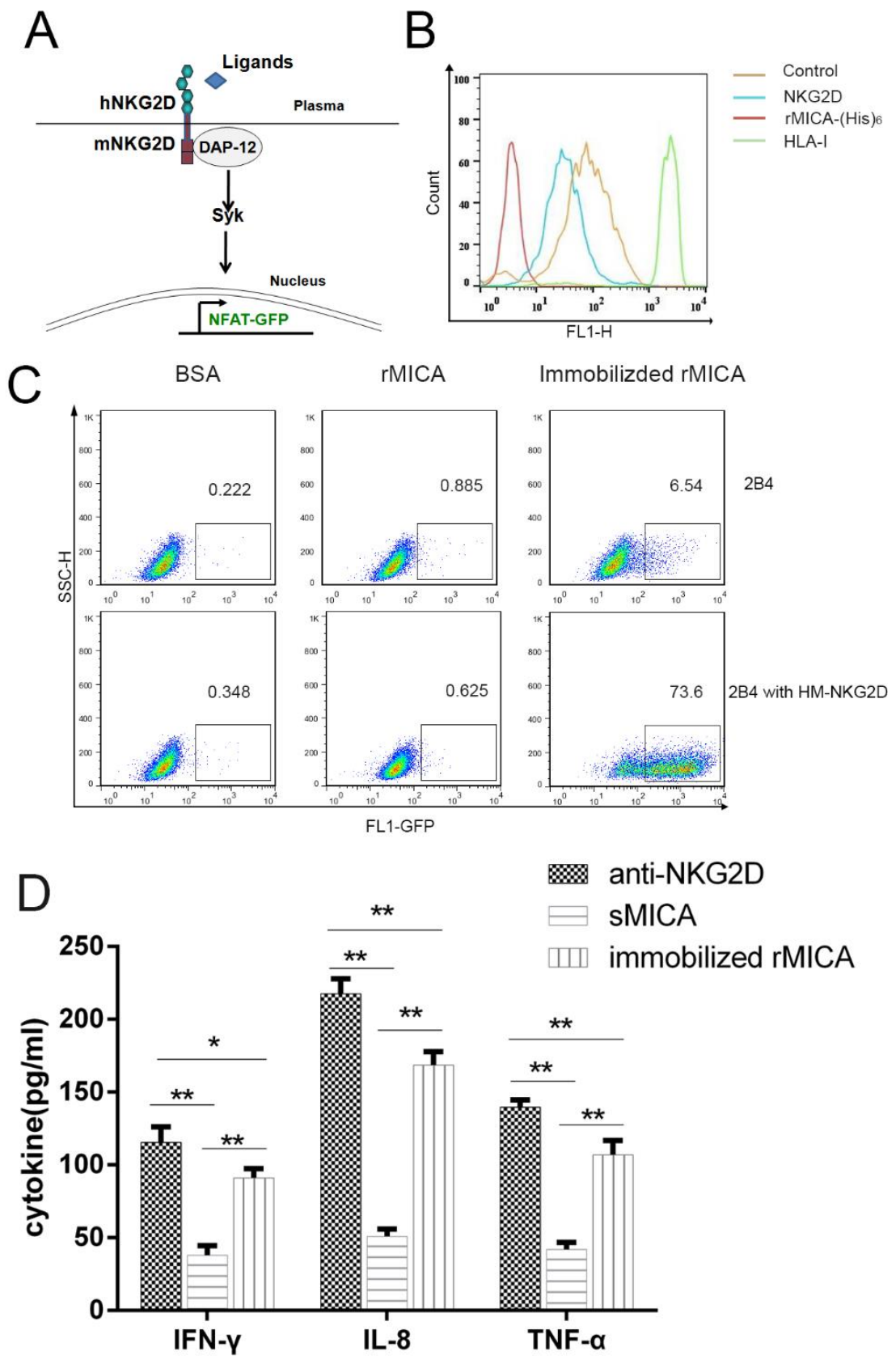

Figure 2. SMICA does not activate the NKG2D receptor pathway. (A) Diagram of NKG2D receptor reporter system. The NKG2D chimeric receptor is an engineered receptor composed of the human NKG2D extracellular domain and the mouse NKG2D membrane-spanning region. Upon binding of ligand to the chimeric NKG2D receptor, downstream signaling results in production of GFP. (B) Flow cytometry analysis of NKG2D2B4 or control cells (2B4 cells) incubated with anti-NKG2D monoclonal antibody, anti-His tag monoclonal antibody, antiHLA-I monoclonal antibody (w6/32), or mouse isotype IgG. (C) Percentage of $\mathrm{GFP}^{+}$cells in NKG2D-2B4 and 2B4 cells incubated with BSA or soluble rMICA *004 or incubated in wells pre-coated with rMICA. (D) The cytokine responses of NK cells to anti-NKG2D monoclonal antibody coated (anti-NKG2D), soluble rMICA, and immobilized rMICA after 24 hours. The supernatants were collected and concentrations of IFN- $\gamma$, TNF- $\alpha$, and IL-8 were determined ( $\mathrm{pg} / \mathrm{ml})$. Plotted are results of triplicate experiments.

After 24 hours of the incubation of 2B4 and NKG2D2B4 cells with NKG2D ligands, flow cytometry was used to detect the percentage of $\mathrm{GFP}^{+}$cells, which is indicative of ligand binding to the chimeric receptor and NKG2D pathway activation. In samples treated with $\mathrm{rMICA}^{*} 004$ or with the control BSA, fewer than $1 \%$ of cells were $\mathrm{GFP}^{+}$(Fig. 2C). The results showed that soluble rMICA*004 did not induce production of GFP. However, in cells incubated with immobilized rMICA, NKG2D receptor-mediated signaling was induced with $73.6 \%$ of cell expressing GFP. Less than $6.25 \%$ of $2 \mathrm{~B} 4$ cells, which

do not express the chimeric receptor but do encode GFP, were fluorescent after 24 hours (Fig. 2C; $\mathrm{t}=10.927$, P < $0.001)$.

Activation of the NKG2D receptor pathway in NK cells should induce production of cytokines. Therefore, we measured cytokines released from NKL cultured in the presence of a monoclonal antibody, or soluble rMICA and immobilized-rMICA. After 24 hours, cell culture supernatants were collected and analyzed with the cytokine Luminex array. The amounts of IFN- $\gamma$, TNF- $\alpha$, and IL- 8 released upon stimulation with immobilized rMICA were 
significantly higher than levels in the presence of soluble rMICA (P < 0.001, Fig. 2D), and other 14 cytokines were not increased. Amounts of cytokines released from NKL cultured with immobilized rMICA were similar to amounts released in the presence of the positive control with anti-NKG2D monoclonal antibodies coated (Fig. 2D).

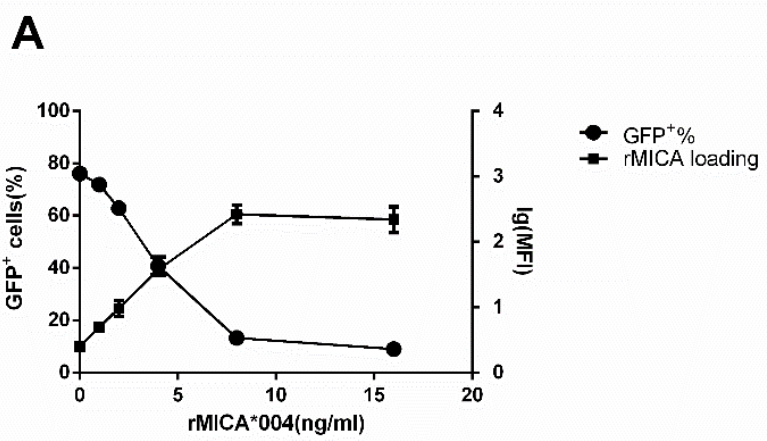

B
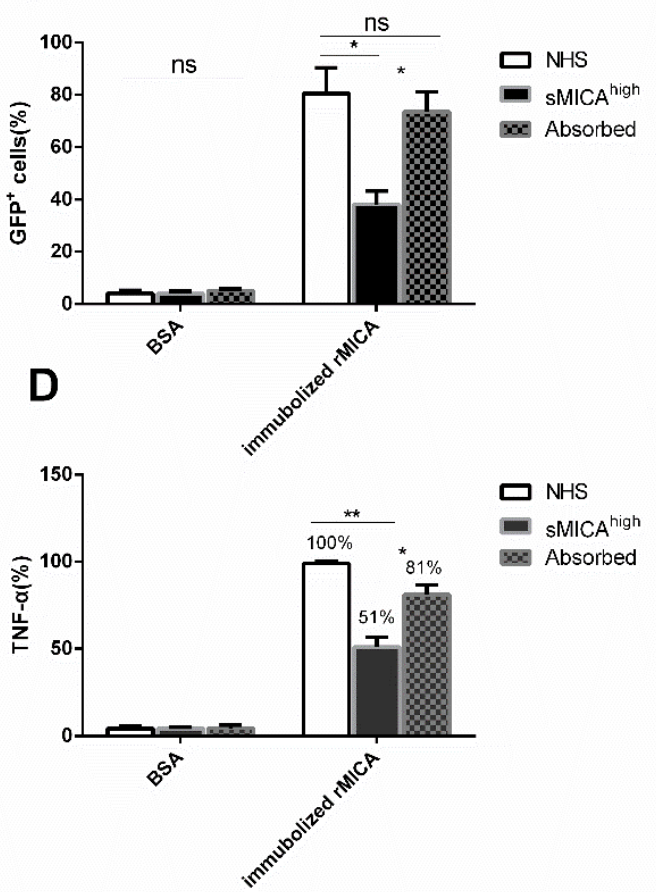

C
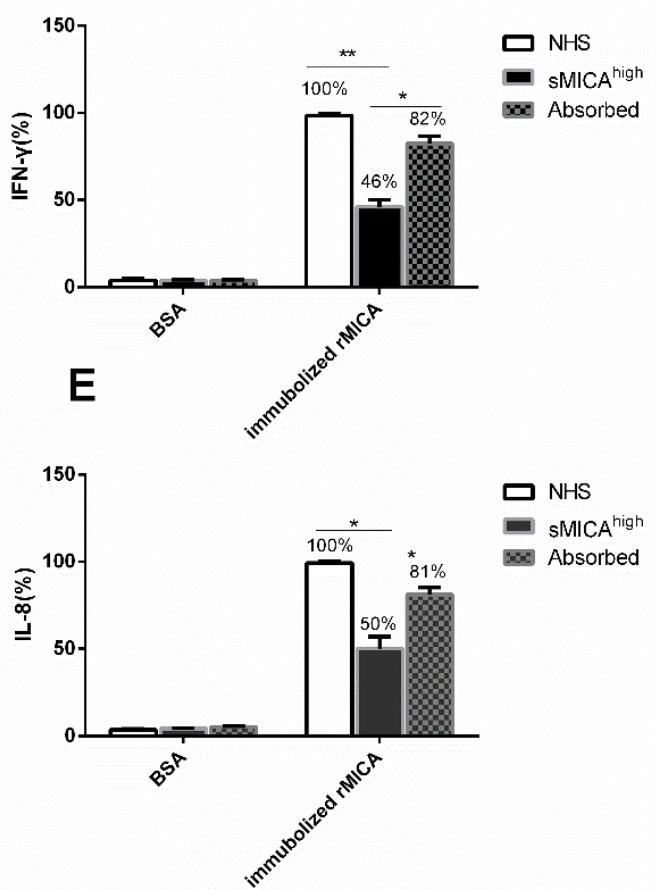

Figure 3. Soluble MICA molecule blocks the activation of NKG2D pathway. (A) Binding of soluble rMICA*004His to NKG2D-2B4 cells detected by staining of the PE-conjugated anti-His-tag antibody. The log of mean fluorescence intensity $(\lg [\mathrm{MFI}])$ as a function of rMICA*004-His is plotted on the right-hand axis. The blocking effect on NKG2D pathway signaling was determined by analysis of $\mathrm{GFP}^{+}$cells; the percent $\mathrm{GFP}^{+}$cells in the presence of each concentration of $\mathrm{rMICA}^{*} 004-\mathrm{His}$ is plotted on the left-hand axis. (B) Percentage of $\mathrm{GFP}^{+}$cells in the presence of sMICA ${ }^{\text {high }}$ patient sera $(\mathrm{N}=10)$, the same sera pre-treated with anti-MICA mAb6B3 beads (Absorbed, N=10), and sera from healthy volunteers (NHS, $\mathrm{N}=10)$. * indicates statistically significant difference $(\mathrm{P}<0.05)$. (C-E) Cytokines (C) IFN- $\gamma$, (D) TNF- $\alpha$, and (E) IL-8 released from NKL incubated with immobilized rMICA. The concentrations of cytokines in supernatant of samples treated with NHS were defined as $100 \%$. The ratio of sample treated with sMICA $^{\text {high }}$ and Absorbed are given. * Indicates statistically significant difference $(\mathrm{P}<0.05)$.

\section{sMICA blocks activation of NKG2D receptor-mediated signaling}

An analysis of the geometrical mean of fluorescence intensity $(\lg [\mathrm{MFI}])$ was used to evaluate the effect of
sMICA and immobilized rMICA on NKG2D report system. Binding increased as a function of soluble rMICA added (Fig. 3A). Conversely, the percentage of $\mathrm{GFP}^{+}$cells decreased with increasing concentration of sMICA added (Fig. 3A). We then evaluated the effect of sera from 
sMICA ${ }^{\text {high }}$ HCC patients and found that addition of patient sera dramatically reduced the percentage of $\mathrm{GFP}^{+}$cells compared to normal health serum (NHS, $t=5.557$, $\mathrm{P}=0.031$, Fig. 3B). In the patient serum depleted of sMICA by absorption with an anti-MICA mAb6B3 beads, the percentage of $\mathrm{GFP}^{+}$cells was near NHS control levels $(t=5.433, p=0.032$, Fig. 3B). These results support the hypothesis that SMICA inhibits activation of the NKG2D pathway.
We next evaluated cytokine release from cultured NKL incubated with immobilized-rMICA in presence of sera from healthy volunteers (NHS), sera from HCC patients wth high level of sMICA (sMICA $\left.{ }^{\text {high }}\right)$, sMICA ${ }^{\text {high }}$ sera depleted of sMICA (Absorbed). We found that IFN$\gamma$, TNF- $\alpha$, and IL- 8 release was inhibited by in presence of sMICA compared to levels from cells cultured in the presence of NHS (Fig. 3C-E). Moreover, the effect was reversed when SMICA was removed from the serum by anti-MICA mAb6B3 beads (Fig. 3C-E).
A

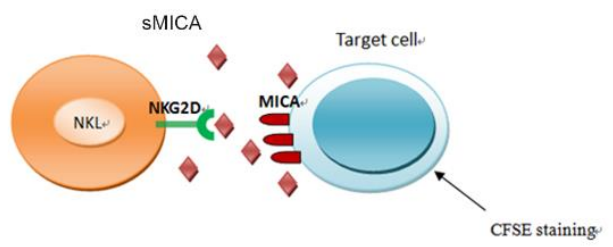

C

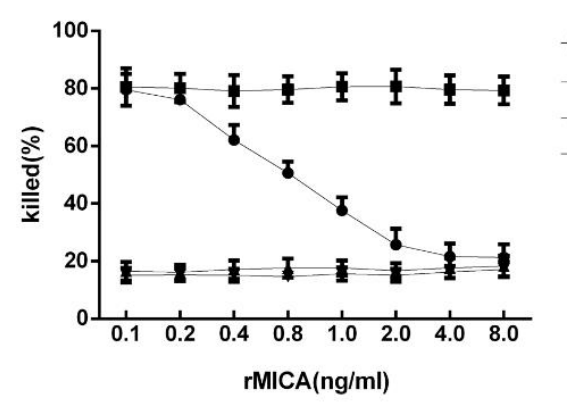

B

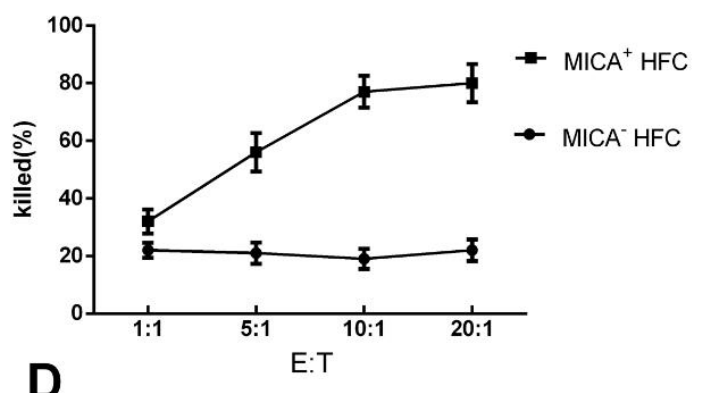

D
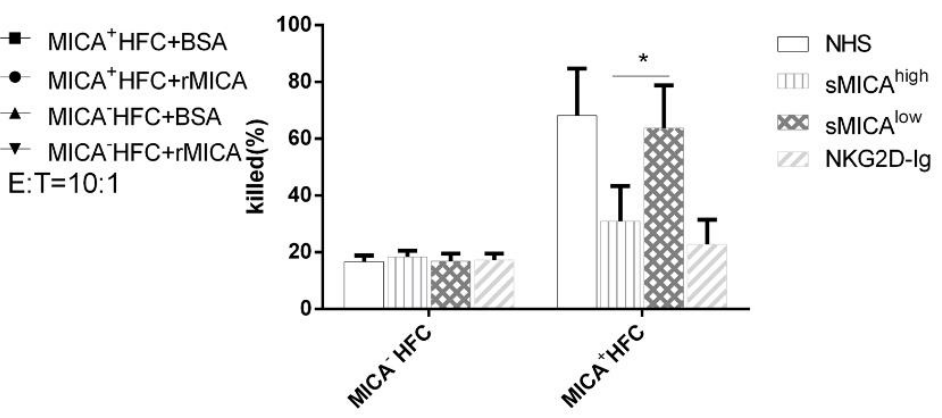

Figure 4. sMICA inhibits the cytotoxicity of NK cells toward MICA ${ }^{+}$target cells. (A) Schematic of components involved in the MICA-NKG2D pathway. NK effector cells were incubated with $\mathrm{MICA}^{+}$target cells stained with CSFE dye. sMICA interferes with cell killing mediated by NK cells. (B) Target cells (5000 cells per assay) with (MICA $\left.{ }^{+} \mathrm{HFC}\right)$ or without MICA expressed human fibroblasts (MICA- HFC) were co-cultured with NKL at different E: T ratios. The mean percentages of dead cells from three replicate experiments are plotted. (C) The percent dead target cells in the presence of soluble rMICA, BSA at finally concentrations diluted from 0.1 to $8.0 \mathrm{ng} / \mathrm{ml}$ at E: T of 10:1. Plotted are means of triplicate experiments. (D) The percent dead target cells in the presence of serum from sMICA ${ }^{\text {high }}$ patients $(\mathrm{N}=10)$ and from sMICA ${ }^{\text {low }}$ patients $(\mathrm{N}=10)$ at $\mathrm{E}: \mathrm{T}$ of 10:1. Normal health serum (NHS) was used as negative control and soluble NKG2D-Ig at concentration at $1.0 \mu \mathrm{g} / \mathrm{ml}$ were used as maximum blocking controls. Plotted are means of three experiments for each tested (C and $\mathrm{D})$.

\section{sMICA reduces the cytotoxicity of NK cells}

NK cells that express NKG2D receptors on the cell surface kill MICA-expressing target cells as we demonstrated previously [11]. To monitor the effect of sMICA on NK cell-mediate cytotoxicity, we incubated target cells, labeled with CSFE, with NKL, stained dead cells with 7-AAD dye, and analyzed the samples using by flow cytometry (Fig. 4A). As target cells, we used human fibroblasts engineered to stably express high levels of MICA*027 membrane proteins and fibroblasts that do not express MICA. The killing efficiency was measured at ratios of effector (NKL) to the target cells $(\mathrm{E}: \mathrm{T})$ of $1: 1$, $5: 1,10: 1$, and 20:1. Killing by NK cells was dependent on the NKG2D-MICA pathway as fibroblasts that do not express MICA were used as the control; the optimal E:T ratio was 10:1 (Fig. 4B).

Both soluble rMICA and BSA interfered with NK cellmediated toxicity toward fibroblasts that express MICA in the immobilized rMICA stimulating system. In the presence of soluble rMICA and at an E: T of 10:1, we observed that the ratio of MICA+ target cell death were 
reduced from $79.5 \%$ to $21.3 \%$ as in the presence of the concentration of rMICA increased from 0.1 to $8.0 \mathrm{ng} / \mathrm{ml}$, But with BSA concentration increased, the ratio of $\mathrm{MICA}^{+}$target cells were remained at the level of $80 \%$ without any dropping. Using MICA- HFC as target cells, there were only about $16 \%$ dead cells observed, in spite of in presence of rMICA or BSA in the same concentrations as above. (Fig. 4C). In a similar experiment, the maximum blocking effect of NKL on the cytotoxicity of $\mathrm{MICA}^{+}$ HFC cells was obtained in presence of NKG2D-Ig fusion protein at concentration above $1.0 \mu \mathrm{g} / \mathrm{ml}$, and normal health serum used as negative blocking control (Fig. 4D). We tested the effects of sera from the sMICA $^{\text {high }}$ and sMICA $^{\text {low }} \mathrm{HCC}$ patient groups on the killing of $\mathrm{MICA}^{+}$ HFC and MICA $^{-}$HFC by NKL in the immobilized rMICA stimulating system. At 1:2 dilution of sMICA $^{\text {high }}$ sera $(n=10)$, there were $30.8 \% \pm 10.1 \%$ of the MICAexpressing target cells were killed compared with $63.7 \% \pm 12.2 \%$ of the target cells killed in presence of $1: 2$ dilution of sMICA $^{\text {low }}$ sera $(n=10)$ from HCC (Fig. 4D); More cells were escaped from killing in presence of sMICA $^{\text {high }}$ serum than that of $\mathrm{sMICA}^{\text {low }}$ serum $(\mathrm{t}=2.919$, $\mathrm{P}=0.043)$. There was no difference observed when used $\mathrm{MICA}^{-} \mathrm{HFC}$ as the target cells, suggesting that high level of sMICA in serum of HCC patient might protect hepatocellular carcinoma from host immune response.

\section{DISCUSSION}

HCC is the most common primary liver cancer, accounting for $90 \%$ of all liver cancers. The tumor cells escape immune response by T lymphocytes and NK cells in various ways $[30,31]$. Recent studies have shown that the mechanisms of immune escape involve activation of immunosuppressive receptors that negatively regulate the immune response and down-regulate host immune clearance of cancerous tissues [32] and the inhibition of activating receptors generally reduces the immune response [33]. Activation receptor NKG2D on NK and T cells is involved in anti-tumor immune responses [34]. Here we showed that a high level of soluble sMICA, an NKG2D ligand, in sera of HCC patients was associated with poor outcome. Wan and his team [35] reported that MICA is released into circulation due to shedding of MICA from cell membranes during tumor transformation or upon stress. We showed that sMICA binds to cells expressing the NKG2D receptor but does not activate NKG2D receptor-mediated signaling and does not activate of NK cells [25]. It was previously reported that the binding of sMICA decreases the expression of NKG2D receptor in NK cells [36]. Zou and co-workers reported that high level of serum sMICA is associated with biliary cast syndrome after liver transplantation [37].
Using NKG2D reporter cells, we showed that the soluble rMICA did not activate the NKG2D pathway, although it bound to the NKG2D-2B4 reporter cells. In contrast, when we incubated the NKG2D-2B4 reporter cells with immobilized rMICA, $73.6 \%$ of cells fluoresced, indicating active NKG2D signaling, after 16 hours of culture. We also demonstrated that NKG2D pathway activation in NK cell lines with immobilized rMICA, but this activation of NKG2D could be blocked by soluble recombinant MICA molecules and by serum from HCC patients that expressed high levels of soluble MICA. Interestingly, immobilized rMICA also induced release of cytokines from NKL cells. These experiments are used as the well-model to understand how sMICA inhibits the immuno-response of NK and CD8+ T cells to tumor cells. Using microscopy, we previously observed that the single NKG2D dimer is not activated until multiple NKG2D dimers gather into a bundle [25]. This aggregation promotes DAP12 phosphorylation and downstream signaling. Soluble MICA does not induce NKG2D receptor aggregation and there is nothing of NKG2D signaling. The performance of this engineering receptor of NKG2D was confirmed by the natural NKG2D receptor of NKL cells.

Since NKG2D receptor is composed of two identical NKG2D molecules, multiple NKG2D receptors might be formed into agglomeration on cell surface when NKG2DLs bind. The immobilized rMICAs can bind and aggregate NKG2D receptors. Therefore, culturing NK cells with immobilized initiates NKG2D receptor pathway. In the study, we are first to use the immobilized rMICA to activate NK cell lines, instead of using MICAexpressing cells. Cytokines released by NKL reflected NKL activation. We found that IFN- $\gamma$, TNF- $\alpha$ and IL-8 were released increasely when NKLs were stimulated by the immobilized rMICA. Our results demonstrated the blocking effect of sMICA on NKL activation in both fluorescence reporter system and cytokine releasing.

In order to further validate that serum sMICA blocks killing of tumor cells by NK cells, we used human fibroblasts that stably express MICA as the target cells. In the absence of added sMICA, about $80 \%$ of MICAexpressing fibroblasts were killed at an E:T of 10:1. However, in presence of sMICA added, either the soluble rMICA or soluble MICA in sera of SMICA $^{\text {high }} \mathrm{HCC}$ patient, the killing of fibroblasts that express MICA was significantly decreased. Since the test for NKG2D pathway activation are based on the immobilized rMICA stimulation, using MICA-expressed cells as the target may be complicate because of many membrane proteins on cell surface. The inhibition of NK cytotoxicity to the target cells can be reversed when serum sMICA is removed (Fig. 3C-E). 
It was previously reported that the formation of sMICA is associated with the MICA*008 allele [38], which produces a truncated protein. This short MICA molecule may be more readily released by exosomes from the cell membrane than the wild-type version [39], most other allelic MICA variants are released by proteolytic shedding $[18,19]$. No such association with MICA*008 mutations were observed in our cohort (data not shown). A limitation of this study was that we did not evaluate other NKG2D ligands, such as SMICB and sULBP, which are potentially present in serum of HCC patients [40, 41]. In a future study, we will screen for all known soluble NKG2D ligands in sera of HCC patients.

In conclusion, this study demonstrated that high levels of serum MICA are correlated with poor prognosis in HCC cases. Our in vitro and cell-based experiments suggest that soluble MICA mediates tumor immune escape through blocking of the NKG2D signaling pathway. This analysis first demonstrated that the blocking effect of serum sMICA on the NKG2D pathway activation. Our findings indicate that sMICA potentially functions as a biomarker for identification of HCC patients at high risk, and also can provide a novel approach for tumor immunotherapy.

\section{Acknowledgments}

We would like to acknowledge grant support from the National Natural Science Foundation of China (grant numbers 81571562, and 81873875) and from the Hunan Science and Technology Project Foundation (2018 JJ2549).

\section{Conflict of interest}

The authors have no conflicts of interest to declare.

\section{References}

[1] Nolte-'t Hoen, EN, CR Almeida, NR Cohen, S Nedvetzki, $\mathrm{H}$ Yarwood, et al. (2007). Increased surveillance of cells in mitosis by human NK cells suggests a novel strategy for limiting tumor growth and viral replication. Blood, 109: 670-673.

[2] Dai, KZ, JC Ryan, C Naper, JT Vaage (2018). Identification of MHC Class Ib Ligands for Stimulatory and Inhibitory Ly49 Receptors and Induction of Potent NK Cell Alloresponses in Rats. J Immunol, 200: 28472859.

[3] Jelencic, V, M Sestan, I Kavazovic, M Lenartic, S Marinovic, et al. (2018). NK cell receptor NKG2D sets activation threshold for the NCR1 receptor early in NK cell development. Nat Immunol, 19: 1083-1092.

[4] Champsaur, M,LL Lanier (2010). Effect of NKG2D ligand expression on host immune responses. Immunol
Rev, 235: 267-285.

[5] Groh, V, R Rhinehart, J Randolph-Habecker, MS Topp, SR Riddell, et al. (2001). Costimulation of CD8alphabeta $T$ cells by NKG2D via engagement by MIC induced on virus-infected cells. Nat Immunol, 2: 255-260.

[6] Jamieson, AM, A Diefenbach, CW McMahon, N Xiong, JR Carlyle, et al. (2002). The role of the NKG2D immunoreceptor in immune cell activation and natural killing. Immunity, 17: 19-29.

[7] Verneris, MR, M Karimi, J Baker, A Jayaswal, RS Negrin (2004). Role of NKG2D signaling in the cytotoxicity of activated and expanded CD8+ T cells. Blood, 103: 30653072.

[8] Bacon, L, RA Eagle, M Meyer, N Easom, NT Young, et al. (2004). Two human ULBP/RAET1 molecules with transmembrane regions are ligands for NKG2D. J Immunol, 173: 1078-1084.

[9] Zwirner, NW, MA Fernandez-Vina, P Stastny (1998). MICA, a new polymorphic HLA-related antigen, is expressed mainly by keratinocytes, endothelial cells, and monocytes. Immunogenetics, 47: 139-148.

[10] Groh, V, J Wu, C Yee, T Spies (2002). Tumour-derived soluble MIC ligands impair expression of NKG2D and T-cell activation. Nature, 419: 734-738.

[11] Zou, Y, W Bresnahan, RT Taylor, P Stastny (2005). Effect of human cytomegalovirus on expression of MHC class I-related chains A. J Immunol, 174: 3098-3104.

[12] Lanier, LL (2015). NKG2D Receptor and Its Ligands in Host Defense. Cancer Immunol Res, 3: 575-582.

[13] Ghadially, H, L Brown, C Lloyd, L Lewis, A Lewis, et al. (2017). MHC class I chain-related protein A and B (MICA and MICB) are predominantly expressed intracellularly in tumour and normal tissue. Br J Cancer, 116: 1208-1217.

[14] Moretta, A, C Bottino, M Vitale, D Pende, C Cantoni, et al. (2001). Activating receptors and coreceptors involved in human natural killer cell-mediated cytolysis. Annu Rev Immunol, 19: 197-223.

[15] Takaki, R, SR Watson, LL Lanier (2006). DAP12: an adapter protein with dual functionality. Immunol Rev, 214: 118-129.

[16] Wang, T, F Sun, Y Wang, J Jiang, M Pan, et al. (2018). NKG2D Immunoligand rG7S-MICA Enhances NK Cellmediated Immunosurveillance in Colorectal Carcinoma. J Immunother, 41: 109-117.

[17] Cao, W, X Xi, Z Hao, W Li, Y Kong, et al. (2007). RAET1E2, a soluble isoform of the UL16-binding protein RAET1E produced by tumor cells, inhibits NKG2D-mediated NK cytotoxicity. J Biol Chem, 282: 18922-18928.

[18] Salih, HR, HG Rammensee, A Steinle (2002). Cutting edge: down-regulation of MICA on human tumors by proteolytic shedding. J Immunol, 169: 4098-4102.

[19] Waldhauer, I, D Goehlsdorf, F Gieseke, T Weinschenk, M Wittenbrink, et al. (2008), Tumor-associated MICA is shed by ADAM proteases. Cancer Res, 68: 6368-6376.

[20] Sun, D, X Wang, H Zhang, L Deng, Y Zhang (2011). MMP9 mediates MICA shedding in human osteosarcomas. Cell Biol Int, 35: 569-574. 
[21] Chitadze, G, M Lettau, S Luecke, T Wang, O Janssen, et al. (2016). NKG2D- and T-cell receptor-dependent lysis of malignant glioma cell lines by human gammadelta $\mathrm{T}$ cells: Modulation by temozolomide and A disintegrin and metalloproteases 10 and 17 inhibitors. Oncoimmunology, 5: e1093276.

[22] Jiang, X, JF Huang, Z Huo, Q Zhang, Y Jiang, et al. (2012). Elevation of soluble major histocompatibility complex class I related chain A protein in malignant and infectious diseases in Chinese patients. BMC Immunol, 13: 62 .

[23] Huergo-Zapico, L, AP Gonzalez-Rodriguez, J Contesti, E Gonzalez, A Lopez-Soto, et al. (2012). Expression of ERp5 and GRP78 on the membrane of chronic lymphocytic leukemia cells: association with soluble MICA shedding. Cancer Immunol Immunother, 61: 1201-1210.

[24] Kloss, S, N Chambron, T Gardlowski, L Arseniev, J Koch, et al. (2015). Increased sMICA and TGFbetal levels in HNSCC patients impair NKG2D-dependent functionality of activated NK cells. Oncoimmunology, 4: e1055993.

[25] Zou, Y, W Luo, J Guo, Q Luo, M Deng, et al. (2018). NK cell-mediated anti-leukemia cytotoxicity is enhanced using a NKG2D ligand MICA and anti-CD20 scfv chimeric protein. Eur J Immunol, 48: 1750-1763.

[26] Duan, S, W Guo, Z Xu, Y He, C Liang, et al. (2019). Natural killer group 2D receptor and its ligands in cancer immune escape. Mol Cancer, 18: 29.

[27] Frazao, A, L Rethacker, M Messaoudene, MF Avril, A Toubert, et al. (2019). NKG2D/NKG2-Ligand Pathway Offers New Opportunities in Cancer Treatment. Front Immunol, 10: 661.

[28] Deng, M, X Gui, J Kim, L Xie, W Chen, et al. (2018). LILRB4 signalling in leukaemia cells mediates $\mathrm{T}$ cell suppression and tumour infiltration. Nature, 562: 605609.

[29] Ming, Y, J Hu, Q Luo, X Ding, W Luo, et al. (2015). Acute Antibody-Mediated Rejection in Presence of MICA-DSA and Successful Renal Re-Transplant with Negative-MICA Virtual Crossmatch. PLoS One, 10: e0127861.

[30] Lesokhin, AM, TM Hohl, S Kitano, C Cortez, D Hirschhorn-Cymerman, et al. (2012). Monocytic CCR2(+) myeloid-derived suppressor cells promote immune escape by limiting activated CD8 T-cell infiltration into the tumor microenvironment. Cancer Res, 72: 876-886.
[31] Li, T, Y Yang, X Hua, G Wang, W Liu, et al. (2012). Hepatocellular carcinoma-associated fibroblasts trigger NK cell dysfunction via PGE2 and IDO. Cancer Lett, 318: 154-161.

[32] Sun, C, J Xu, Q Huang, M Huang, H Wen, et al. (2017). High NKG2A expression contributes to NK cell exhaustion and predicts a poor prognosis of patients with liver cancer. Oncoimmunology, 6: e1264562.

[33] Bugide, S, MR Green, N Wajapeyee (2018). Inhibition of Enhancer of zeste homolog $2(\mathrm{EZH} 2)$ induces natural killer cell-mediated eradication of hepatocellular carcinoma cells. Proc Natl Acad Sci U S A, 115: E3509E3518.

[34] Sheppard, S, J Guedes, A Mroz, AM Zavitsanou, H Kudo, et al. (2017). The immunoreceptor NKG2D promotes tumour growth in a model of hepatocellular carcinoma. Nat Commun, 8: 13930.

[35] Zingoni, A, E Vulpis, F Cecere, MG Amendola, D Fuerst, et al. (2018). MICA-129 Dimorphism and Soluble MICA Are Associated With the Progression of Multiple Myeloma. Front Immunol, 9: 926.

[36] Jia, HY, JL Liu, MZ Yuan, CJ Zhou, WD Sun, et al. (2015). Regulation Roles of MICA and NKG2D in Human Renal Cancer Cells. Asian Pac J Cancer Prev, 16: 3901-3905.

[37] Zou, Y, X Yang, X Jiang, H Wang, Q Hao, et al. (2009). High levels of soluble Major Histocompatibility Complex class I related chain A (MICA) are associated with biliary cast syndrome after liver transplantation. Transpl Immunol, 21: 210-214.

[38] Jiang, X, Y Zou, Z Huo, P Yu (2011). Association of major histocompatibility complex class I chain-related gene A microsatellite polymorphism and hepatocellular carcinoma in South China Han population. Tissue Antigens, 78: 143-147.

[39] Ashiru, O, P Boutet, L Fernandez-Messina, S AgueraGonzalez, JN Skepper, et al. (2010). Natural killer cell cytotoxicity is suppressed by exposure to the human NKG2D ligand MICA*008 that is shed by tumor cells in exosomes. Cancer Res, 70: 481-489.

[40] Huang, B, R Sikorski, P Sampath, SH Thorne (2011). Modulation of NKG2D-ligand cell surface expression enhances immune cell therapy of cancer. J Immunother, 34: 289-296.

[41] Sharma, N,MA Markiewicz (2019). Constitutive expression of ULBP-4 on monocytes regulates NK cell NKG2D expression. Blood Adv, 3: 1563-1567. 